\title{
Cocaine Abstinence During Methadone Maintenance: Effects of Repeated Brief Exposure to Voucher-Based Reinforcement
}

\author{
Stacey C. Sigmon \\ Johns Hopkins University School of Medicine
}

\author{
Christopher J. Correia \\ Auburn University
}

\author{
Maxine L. Stitzer \\ Johns Hopkins University School of Medicine
}

\begin{abstract}
This study determined whether abstinence engendered by intermittent reinforcement might generalize to nonreinforced periods and enhance overall rates of cocaine abstinence among methadone maintenance patients. Participants were randomized to 1 of 3 groups. The quantitative group $(n=14)$ earned incentives for a $50 \%$ decrease in urine benzoylecgonine concentrations; the qualitative group $(n=13)$ earned incentives for providing urines with concentrations $<300 \mathrm{ng} / \mathrm{ml}$. Both reinforced groups received 12 random opportunities to earn $\$ 100$ in vouchers for abstinence. The control group $(n=15)$ was encouraged to abstain on 12 occasions under the same schedule. Incentive participants achieved significantly more cocaine abstinence on earning than on nonearning days, with no difference between quantitative and qualitative groups. The study supports brief abstinence test efficacy but did not find that unpredictable opportunities to earn incentives generalized to nonreinforced days.
\end{abstract}

A significant proportion of patients continue to abuse drugs during methadone treatment (Grella, Anglin, \& Wugalter, 1997; Kidorf \& Stitzer, 1993), and effective interventions for reducing cocaine use during methadone maintenance treatment could significantly improve outcomes for that modality. Contingency management interventions, using voucher-based incentives contingent on drug abstinence, have proven quite efficacious for improving cocaine use outcomes in both outpatient psychosocial treatment (Higgins et al., 1991, 1993, 1994) and methadone maintenance treatment (Silverman et al., 1996, 1998). Nevertheless, in the methadone setting these interventions are usually effective (i.e., producing long periods of continuous cocaine abstinence) with only 50\%-60\% of cocaine-abusing methadone patients, whereas the remainder of patients continue to abuse cocaine during treatment (Silverman et al., 1996).

Our group has been interested in exploring the parameters of voucher reinforcement procedures in order to develop an

Stacey C. Sigmon and Maxine L. Stitzer, Department of Psychiatry and Behavioral Sciences, Johns Hopkins University School of Medicine; Christopher J. Correia, Department of Psychology, Auburn University.

This study was supported by Research Grant DA12439 and Training Grant T32DA07209 from the National Institute on Drug Abuse. We thank Liam Harmon, Jacalyn Smith, and Michael Sklar for their assistance in implementing the treatment and collecting the research data. We also thank Tim Mudric, Paul Nuzzo, and Laura Pennington for their assistance in managing and summarizing the data.

Correspondence concerning this article should be addressed to Stacey C. Sigmon, who is now at the Department of Psychiatry, University of Vermont, 1 South Prospect Street, UHC 1415, Burlington, VT 05401. E-mail: stacey.sigmon@uvm.edu intervention that might maximize initial response to contingency management interventions and therefore allow the largest possible proportion of patients to reap the benefits of abstinence incentive programs. Toward this end, we have developed a brief abstinence reinforcement procedure that seeks to maximize patients' response to incentives by offering a relatively high magnitude reinforcer $(\$ 100)$ for a low-response requirement (i.e., 2 days of cocaine abstinence; Robles et al., 2000). Such brief durations of cocaine abstinence can be detected using quantitative urine testing in which abstinence is defined as a $50 \%$ or greater reduction over 2 days in urine benzoylecgonine concentration (Preston, Silverman, Schuster, \& Cone, 1997). In the more conventional qualitative urinalysis testing approach, the benzoylecgonine concentration must be below $300 \mathrm{ng} / \mathrm{ml}$ to be considered negative, and participants may need to abstain from cocaine use for 2-5 days to produce a negative urine specimen. Quantitative testing is more sensitive and enables differentiation between "new" cocaine use and carryover from previous uses. This increased sensitivity reduces the delay between initiation of cocaine abstinence and reinforcement delivery, thereby enabling a more accurate implementation of the reinforcement contingencies (i.e., fewer instances of unreinforced abstinence) and possibly enhancing treatment efficacy (Preston, Umbricht, Wong, \& Epstein, 2001).

One previous study examining the effects of a brief (2-day) abstinence test on cocaine use among methadone patients showed efficacy of the procedure in that $84 \%$ of participants met the abstinence criteria during the test week compared with $34 \%-36 \%$ during the preceding or following weeks, when no abstinence reinforcement contingency was in place (Robles et al., 2000). In another study (Katz et al., 2002), approximately $70 \%$ of participants met the criteria 
for brief abstinence initiation, and this response was stable across three randomly ordered study conditions. These results suggested that such procedures might offer an effective approach to maximizing the proportion of patients who initiate abstinence. Given the remarkably high percentage of participants who responded favorably to the brief abstinence test in previous studies, we were interested in whether periodic, repeated exposures to a brief abstinence test might reduce overall rates of cocaine use through a carryover effect in which abstinence generalized to nonreinforced time periods.

It is a well-known principle of operant conditioning that an intermittent, unpredictable schedule of reinforcer delivery (e.g., a variable interval schedule) can produce stable, persistent behavior (Skinner, 1953). Thus, unpredictable presentation of earning opportunities might produce sustained cocaine abstinence, even on days when abstinence incentives are not available, owing to patients' anticipation of the next, unpredictable earning opportunity. If abstinence did generalize to nonreinforced time periods (as evidenced by increased overall rates of cocaine abstinence among voucher groups compared with the nonreinforced control group), the repeated brief abstinence protocol could be a cost-effective way to deliver abstinence incentives in community treatment programs.

Thus, the main purpose of the present study was to examine the likelihood of abstinence generalization from days on which cocaine abstinence incentives were available to days during which no incentives were available. Toward this end, periodic brief abstinence tests were scheduled under an unpredictable schedule once within each 2-week time block during the study. During each brief abstinence test, participants could earn $\$ 100$ in vouchers if cocaine abstinence was detected, as determined by either quantitative or qualitative urine testing criteria. Whereas quantitative testing may facilitate immediate reinforcement of brief abstinence, qualitative testing is the method commonly used in community clinics and is an approach that could be more feasibly implemented in community programs should it prove to enhance rates of cocaine abstinence compared with rates observed in a control group that did not receive any abstinence reinforcement.

Use of the two urinalysis methods (quantitative and qualitative) to test the main study hypothesis provided the opportunity to directly examine how these testing methods compare in their sensitivity to detect cocaine abstinence and consequent ability to provide accurate and immediate reinforcement for brief periods of abstinence. On the basis of the work of Preston and colleagues (Preston et al., 2001), it was anticipated that the quantitative testing method would be associated with more abstinence detections and higher rates of abstinence reinforcement than qualitative testing.

A final aim of this study was to determine whether the repeated opportunities to earn incentives for achieving brief periods of abstinence would enhance participants' subsequent success when the contingencies required sustained abstinence for reinforcement. Toward this end, all participants received a final abstinence posttest at the end of the 6-month intervention during which they could earn up to
$\$ 500$ if they achieved 2 weeks of continuous cocaine abstinence. Again, on the basis of results of the Preston et al. (2001) study, it was hypothesized that those subjects initially reinforced for cocaine abstinence under the quantitative testing method would exhibit higher rates of sustained abstinence than those reinforced under the qualitative criteria because of more prior abstinence experience and/or more prior exposure to reinforcement.

\section{Method}

\section{Participants}

Study participants were 46 cocaine-abusing methadone maintenance patients enrolled between January 2000 and June 2001 at the Behavioral Pharmacology Research Unit at Johns Hopkins University School of Medicine. Participants had been enrolled in the methadone treatment program for an average of $3.3(S D=0.1)$ months, and all had prior experience with voucher incentive procedures. Participants on average were $42.4(S D=1.0)$ years old and had completed $11.6(S D=0.3)$ years of education. Fifty-four percent were African American, and 57\% were male. During the 4-week screening period prior to the study, participants showed high rates of both cocaine ( $M=83 \%$ of urines positive) and opiate ( $M=64 \%$ of urines positive) use.

\section{Standard Treatment}

Methadone maintenance treatment included daily methadone (100 mg/day) and $2 \mathrm{hr}$ per week of counseling ( $1 \mathrm{hr}$ individual, 1 hr group). Two participants requested and were given dose reductions (to $65 \mathrm{mg}$ and $75 \mathrm{mg}$, respectively) during the study. All participants provided a urine specimen three times per week. Take-home methadone doses were allowed only on holidays and for emergencies. Patients were terminated from treatment and from the study if they missed 3 consecutive dosing days. Upon completion of the study, 10 participants who had entered short-term research slots underwent a 90-day methadone detoxification during which their methadone dose was gradually reduced to $0 \mathrm{mg} ; 36$ participants were able to remain in long-term treatment at the research clinic. Detoxing participants were encouraged to continue methadone treatment elsewhere and were assisted with finding alternative treatment during this time.

\section{Urine Collection and Testing}

Urine samples were collected every Monday, Wednesday, and Friday under observation of a trained technician and were immediately temperature tested to confirm their validity. If a urine specimen measuring above $37.2^{\circ} \mathrm{C}\left(99^{\circ} \mathrm{F}\right)$ or below $34.4{ }^{\circ} \mathrm{C}$ $\left(94^{\circ} \mathrm{F}\right)$ was submitted, the sample was discarded and the participant was required to leave a second specimen. Participants who failed to deliver a urine specimen on a scheduled collection day were required to do so on the following day; however, missing specimens were considered positive for purposes of determining voucher earnings and for subsequent analyses. All urine specimens were tested for cocaine and opiates using an on-site Toshiba 30R system (Syva-Dade Behring, San Jose, CA).

\section{Experimental Procedures}

Prestudy screening period. During the 4 weeks prior to the study, patients attended the clinic on Monday, Wednesday, and 
Friday to provide urine samples. During this period, urinalysis results were monitored to identify patients with continued cocaine use. Patients were considered eligible for study participation if 3 out of the past 10 and 2 out of the past 6 urine samples they provided during the screening period were positive for cocaine. Patients were blind to these eligibility criteria. Forty-six patients met eligibility criteria and were enrolled in the study.

Stratification and random assignment. At the beginning of the study, participants were stratified and randomly assigned to the quantitative $(n=14)$, qualitative $(n=16)$, or control $(n=16)$ condition. Participants were stratified on two baseline characteristics during the 4-week screening period: rate of cocaine-positive urine specimens $(>65 \%$ or $<65 \%)$ and rate of opiate-positive urine specimens $(>25 \%$ or $<25 \%)$. Participants were assigned a binary score ( 0 or 1$)$ on each of these two characteristics, with a total of four possible binary score combinations. For each possible combination, the first participant was randomly assigned to a study condition. The next participant with the same combination of characteristics was randomly assigned to one of the remaining two groups. The third participant was assigned to the remaining group. This procedure ensured that participants were randomly assigned but that no single combination of characteristics would be overrepresented in a group. The three final experimental groups did not significantly differ on baseline rates of cocaine- or opiate-positive urine specimens, nor did they differ on any other demographic characteristic.

On the day of randomization, participants were informed of their group assignment and received a printed description of the reinforcement contingencies for their experimental phase, including a description of quantitative versus qualitative urinalysis testing sensitivity. In addition, a staff member explained all relevant study procedures, gave each participant a quiz with feedback to assess his or her level of comprehension of these procedures, and provided corrective feedback as necessary. The quiz-plus-feedback procedure was repeated at the beginning of each of the random earning opportunities $(n=12)$ throughout the study.

Sample attrition. Five participants (1 control, 4 qualitative) failed to complete the study. Four of these participants (1 control, 3 qualitative) terminated participation during the first several weeks of the protocol; given the amount of incomplete data for these participants, they were excluded from the subsequent analyses. Inspection of individual data for the qualitative participants who terminated participation showed that all had earned at least one reinforcer; thus, it does not appear that these dropouts were due to difficulty of abstinence criteria. One qualitative participant completed all but the final week of the study; the three missing samples for that week were interpolated as cocaine positive, and data for this participant were included in all analyses. Thus, a total of 42 participants were considered study completers and used in all subsequent analyses (14 quantitative, 13 qualitative, 15 control).

\section{Treatment Interventions: Phase 1, Main Study}

Quantitative testing condition $(n=14)$. During Weeks 1-24, participants in the quantitative voucher group periodically received incentives contingent on demonstrating brief cocaine abstinence (as evidenced by a $50 \%$ decrease in urinary benzoylecgonine concentrations). Twelve earning opportunities (earning days) were implemented over the 24-week period, using a block randomization strategy such that one out of every six urine test days (i.e., Monday, Wednesday, or Friday) was randomly selected as an earning day. This procedure resulted in approximately one earning opportunity per each 2-week block. At each opportunity, participants were told that they would earn a $\$ 100$ voucher (described below) if their urine-test results on the next urine day indicated that they had abstained from cocaine use. Participants were informed that the urine test would be sufficiently sensitive to identify recent (i.e., 2-day) abstinence and that as long as they discontinued their drug use immediately, they would likely be able to earn the voucher; however, participants were also instructed that they would have the best chance of earning all vouchers if they remained continuously abstinent from cocaine for the entire 24 -week period. On each of the 12 earning days, participants were considered cocaine abstinent if their urinary benzoylecgonine concentration had reduced by $50 \%$ from the previous urine day or was less than $300 \mathrm{ng} / \mathrm{ml}$ (Preston et al., 1997). During the remaining 60 urine test days (nonearning days) of Phase 1, participants provided a urine sample but could not earn vouchers for cocaine abstinence.

Qualitative testing condition $(n=13)$. In the qualitative voucher group, participants received incentives contingent on demonstrating cocaine abstinence (as evidenced by a benzoylecgonine concentration of $300 \mathrm{ng} / \mathrm{ml}$ or less). As with the quantitative group, participants had 12 earning days, randomly distributed throughout the 24-week period on which they could earn $\$ 100$ in vouchers if their urine-test results on the next collection day indicated cocaine abstinence. However, participants were considered cocaine abstinent only if their urinary benzoylecgonine concentration met qualitative criteria for abstinence $(<300 \mathrm{ng} / \mathrm{ml})$. Participants were told at each earning opportunity that they might be able to earn the voucher if they abstained for the next 2 days but that they would have the best chance of earning all vouchers if they remained continuously abstinent from cocaine for the entire 24week period. During the remaining 60 urine test days (nonearning days) of Phase 1, participants provided a urine sample but could not earn vouchers.

Usual care control $(n=15)$. The third experimental group was a control condition in which, on 12 randomly determined days during the 24-week period (encouragement days), participants were encouraged to abstain from using cocaine and told they would meet with a research assistant on their next scheduled urine collection day to discuss their urine-test result. Feedback of urinetest results was the only consequence for cocaine use or abstinence in this condition.

\section{Sustained Abstinence Test: Phase 2, Postintervention Manipulation}

Following completion of the 24-week intervention, participants were exposed to a 2-week washout period (Weeks 25 and 26), during which urine specimen collection continued but no contingencies were placed on cocaine abstinence or use. Immediately following this washout period, all participants (including control participants) received a 2-week abstinence test (Weeks 27 and 28) during which they could earn a maximum of $\$ 500$ in vouchers for evidence of sustained cocaine abstinence (cf. Katz et al., 2002). On the first day of this 2 -week phase (i.e., Monday), participants were instructed that they would earn a $\$ 100$ voucher if they demonstrated that they had abstained from cocaine use between today and the next scheduled urine day (i.e., Wednesday). The quantitative abstinence criteria was used, whereby participants were considered cocaine abstinent if their urinary benzoylecgonine concentration had reduced by $50 \%$ from the previous day or was less than $300 \mathrm{ng} / \mathrm{ml}$. Additional $\$ 100$ vouchers could be earned on Friday of the first week and on Monday, Wednesday, and Friday of the second week if the participant continued to submit urinalysis evidence consis- 
tent with sustained cocaine abstinence. All participants were aware that once they submitted a cocaine-positive urine specimen, vouchers were no longer available for the remainder of Phase 2.

\section{Vouchers}

For the quantitative and qualitative voucher groups during Phase 1, and for all participants during Phase 2, each cocainenegative urine specimen on an earning day was worth $\$ 100$ in vouchers. Once earned, vouchers could not be lost. Voucher earnings could be used to purchase products (clothes, food, appliances, sports equipment, etc.) and pay for services (rent, telephone, insurance, transportation, etc.) available in the community, as long as the purchases were deemed consistent with the participant's treatment goals. Participants were not given cash; all purchases and payments were made directly by the research staff. The average exchange delay was approximately 2 days.

\section{Data Analysis}

For consistent comparison of data across the three experimental groups, all urine results were examined using the quantitative criteria (i.e., each urine test was judged as showing abstinence versus use since the previous urine test according to the criteria of $50 \%$ reduction in benzoylecgonine or $<300 \mathrm{ng} / \mathrm{ml}$ ). The primary hypothesis was examined by comparing mean percentage of cocaine-negative urine specimens across the three experimental groups. Urine data were analyzed in a two-way PROC MIXED repeated measures analysis of variance (ANOVA) that included experimental group (three levels) and earning condition (12 earning or encouragement days vs. 60 nonearning or nonencouragement days). Planned comparisons ( $t$ tests) were conducted to examine relationships between study conditions within and between groups using Tukey's honestly significant difference procedure. To examine whether there was a time trend in abstinence achieved across each of the 12 successive earning opportunities, we also examined urinalysis data using the generalized estimating equation approach.

Sensitivity of the two urine testing methods for detecting and reinforcing abstinence was examined using chi-square and independent $t$ tests to compare (a) the percentage of samples detected as abstinent under quantitative versus qualitative testing methods, (b) the percentage of available vouchers earned by the quantitative versus qualitative groups on the 12 earning opportunities, and (c) the amount of voucher money earned by subjects in the quantitative versus qualitative groups.

In Phase 2, percentage of subjects initiating abstinence and percentage sustaining abstinence through the 2 -week test were calculated for participants in the three groups combined. To test the hypothesis about effects of prior intermittent abstinence reinforcement on sustained abstinence in the final 2-week abstinence test, we compared percentage of urines (out of five) indicating sustained cocaine abstinence across the three groups in one-way ANOVA and in survival analysis. Finally, to examine the relationship between cocaine abstinence during study phases requiring intermittent versus sustained abstinence, we conducted Pearson correlations between percentage of cocaine-negative samples submitted by individual subjects during the 24 -week main intervention and percentage of tests indicating continuous cocaine abstinence (out of five) during the posttest. For these analyses, only data from the two voucher incentive groups were used, and these data were combined.

\section{Results}

\section{Cocaine Abstinence During the 24-Week Intervention Period (Phase 1)}

Earning versus nonearning days. Mean percentage of cocaine-negative samples (as determined by quantitative testing criteria) for the 60 nonearning days and 12 earning (or encouragement) days of Phase 1 is shown in Figure 1 for each of the three experimental groups. Results of PROC MIXED ANOVA revealed a significant effect of earning versus nonearning days but no significant differences across groups on nonearning days, indicating an absence of generalization from earning to nonearning days within the reinforced groups. In post hoc testing, mean percentage of cocaine-negative specimens did not differ significantly between nonearning and encouragement days among control participants (54\% vs. $57 \% ; p=.53)$. In contrast, in the quantitative testing group, mean percentage of cocainenegative specimens was significantly higher on earning than on nonearning days (70\% vs. $57 \% ; p<.01)$. Similarly, in the qualitative group, mean percentage of cocaine-negative samples increased from $47 \%$ on nonearning days to $63 \%$ on earning days $(p<.01)$.

If a significant trend were observed in abstinence across the 12 successive earning days, it might suggest that participants were improving in their ability to achieve cocaine abstinence as a function of repeated exposure to these brief earning opportunities. However, when data were analyzed with a time effect included, no significant effects were observed for group, time, or Time $\times$ Group interaction. The percentage of participants abstinent ranged from 57\% to $93 \%(M=70 \%, S D=11 \%)$ in the quantitative group on each of the 24 earning opportunities and from $46 \%$ to $77 \%$ $(M=63 \%, S D=10 \%)$ in the qualitative group.

Influence of urinalysis testing method. Table 1 shows individual participant results for both quantitative and qualitative urine testing of their urine specimens on the 12 earning days. Although the amount of cocaine abstinence did not differ between the two types of urinalysis testing, the greater sensitivity of the quantitative criteria for detecting short-term abstinence can be seen by the higher percentage of abstinence detections within both groups under quantitative versus qualitative criteria: $70 \%$ and $63 \%$ of urine tests were judged to reflect abstinence under the quantitative criteria, compared with $42 \%$ and $40 \%$ under the qualitative abstinence criteria. Chi-square revealed a significantly higher percentage of abstinence detections under quantitative than under qualitative testing criteria $(p<.01)$. This difference in abstinence detection sensitivity also can be seen in voucher earnings under the two testing conditions. Specifically, the group that received vouchers based on quantitative testing criteria earned $70 \%$ of available vouchers (mean earnings $=\$ 804$ ), whereas the group receiving vouchers based on qualitative criteria earned only $40 \%$ of available vouchers (mean earnings $=\$ 480$ ). Significantly higher voucher earnings for the quantitative versus qualitative criteria were revealed by $t$ test $(p<.05)$. Further, it is notable that three qualitative participants ( $23 \%$ of the group) 


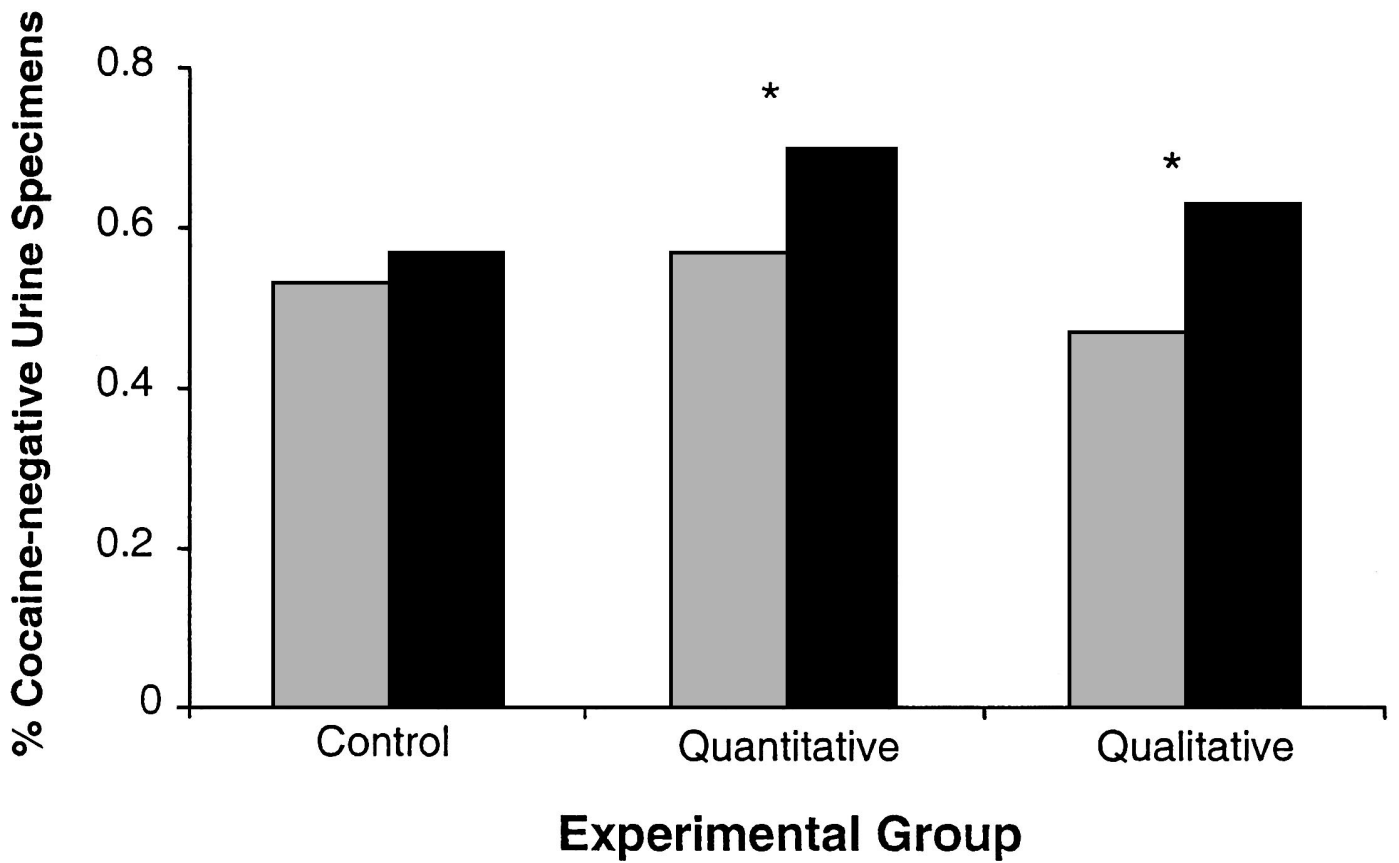

Figure 1. Percentage of cocaine-negative urine specimens (as determined by quantitative testing) provided by the control, quantitative, and qualitative experimental groups during Phase 1. Asterisks indicate significant within-group differences between nonearning and earning days. Gray bars indicate nonearning days; black bars indicate earning/encouragement days.

never earned a reinforcer, whereas all quantitative participants except for one earned at least four reinforcers.

Cocaine abstinence during the postintervention abstinence test (Phase 2). When data were collapsed across the three experimental groups, cocaine abstinence was detected for $75 \%$ of participants over the first 2 days of the intervention (Monday and Wednesday), when the first $\$ 100$ voucher could be earned. Abstinence rates gradually declined over the course of the next 2 weeks, with $38 \%$ of participants abstinent on the final day. Neither the overall mean percent-

Table 1

Individual Participant Performance on Earning Days

\begin{tabular}{|c|c|c|c|c|}
\hline \multirow[b]{2}{*}{ Participant } & \multicolumn{2}{|c|}{ Quantitative group $(N=14)$} & \multicolumn{2}{|c|}{ Qualitative group $(N=13)$} \\
\hline & $\begin{array}{c}\text { Quantitative } \\
\text { criteria }\end{array}$ & $\begin{array}{c}\text { Qualitative } \\
\text { criteria }\end{array}$ & $\begin{array}{c}\text { Quantitative } \\
\text { criteria }\end{array}$ & $\begin{array}{c}\text { Qualitative } \\
\text { criteria }\end{array}$ \\
\hline 1 & 100 & 100 & 100 & 100 \\
\hline 2 & 100 & 100 & 100 & 83 \\
\hline 3 & 100 & 75 & 100 & 83 \\
\hline 4 & 100 & 100 & 83 & 58 \\
\hline 5 & 100 & 100 & 75 & 67 \\
\hline 6 & 92 & 83 & 67 & 17 \\
\hline 7 & 83 & 0 & 58 & 17 \\
\hline 8 & 67 & 0 & 58 & 58 \\
\hline 9 & 58 & 8 & 45 & 27 \\
\hline 10 & 50 & 8 & 42 & 0 \\
\hline 11 & 42 & 0 & 42 & 8 \\
\hline 12 & 42 & 17 & 33 & 0 \\
\hline 13 & 33 & 0 & 17 & 0 \\
\hline 14 & 8 & 0 & & \\
\hline Mean \% & 70 & 42 & 63 & 40 \\
\hline
\end{tabular}

Note. Values are percentages of cocaine-negative urine specimens out of the 12 earning days, presented for each individual participant. To allow visual comparison of the two types of urinalysis testing, results based on both quantitative and qualitative testing are presented. However, voucher earnings for the quantitative group were determined using the quantitative criteria (leftmost column) and earnings for the qualitative group were determined using the qualitative criteria (rightmost column). 
age of cocaine-negative samples indicating sustained cocaine abstinence nor duration of continuous abstinence in survival analysis differed across groups. Mean percentage of cocaine negative samples was $76 \%, 66 \%$, and $52 \%$ for the control, quantitative, and qualitative groups, respectively.

There were significant correlations between the percentage of contiguous cocaine-negative specimens submitted during the 2-week abstinence posttest in Phase 2 and the percentage of cocaine-negative urine specimens previously submitted on both earning days $(r=.74, p=.00)$ and nonearning days $(r=.84, p=.00)$ during Phase 1 .

\section{Discussion}

The present study replicated previous research by demonstrating that the brief abstinence test, which uses a combination of high reinforcer magnitude $(\$ 100)$ and low response requirement (2-3 days of cocaine abstinence), can reliably initiate brief periods of cocaine abstinence in methadone maintenance patients (Figure 1). Data from the control group, in which participants were encouraged to abstain from cocaine but not offered vouchers, provide additional evidence that these increases in cocaine abstinence are a result of the contingent incentives rather than simply instruction and feedback. Rates of abstinence detected with quantitative testing in both study phases $(60 \%-70 \%)$ were generally comparable to rates seen in previous studies using the brief abstinence test (Katz et al., 2002; Robles et al., 2000).

Our hypothesis about response generalization under the intermittent schedule of reinforcement was not supported in the present study. Rates of abstinence observed on nonearning days during Phase 1 were consistently lower than those observed on earning days but not significantly different from control rates for either reinforcement group (Figure 1), suggesting that participants did not stop using cocaine in anticipation of potential upcoming earning opportunities. Although variable interval (VI) schedules have been shown to produce stable, persistent behavior (Skinner, 1953), the schedule used here differed from a standard VI schedule in several ways. First, in contrast to reinforcing a particular active response (e.g., lever pressing), the vouchers in Phase 1 were contingent on demonstrating evidence of cocaine abstinence. That is, this study reinforced the absence of a behavior (recent drug use) rather than reinforcement of an observable response. Intermittent reinforcement of abstinence therefore might not be expected to produce the degree of stable response and generalization typically seen with VI schedules. Second, the sequence or timing of the reinforcers in a VI schedule can influence the subsequent stability and levels of the target behavior. For example, a series of long intervals between reinforcements will likely lower the response rate and result in less consistent responding (Sidman, 1960). In this study, the randomly distributed opportunities occurred on average every 2 weeks. Perhaps reducing the length of the interval between each opportunity (e.g., presenting brief abstinence tests approximately once per week) would have increased generalization of absti- nence to nonearning days. Finally, human drug taking can be influenced by contextual and stimulus control information that is not available to animals. In the present study, for example, the 12 random earning opportunities were preceded by verbal instructions that clearly identified the imminent earning opportunity. Thus, study participants could wait for the instructions before reducing their drug use and, at least in the quantitative group, still earn most or all of the available reinforcers. It is possible that an unannounced, randomly implemented abstinence reinforcement schedule would have produced different results. Nevertheless, the protocol was important to investigate, as it is the sort that might be attractive to community clinics in order to reduce staff burden related to frequent urine monitoring as well as costs of both urine testing and voucher incentive payouts.

Although the type of urinalysis testing (quantitative vs. qualitative) did not significantly influence rates of cocaine abstinence achieved in this study, our data do highlight the differences inherent in the use of quantitative versus qualitative criteria for abstinence testing in relation to reinforcer exposure (Table 1). Specifically, participants in the qualitative group received fewer vouchers despite achieving overall rates of cocaine abstinence that were comparable to those seen in the quantitative group. This observation is consistent with a previous study (Preston et al., 2001) that used quantitative testing during initial stages of a contingency management program to enhance contact with reinforcers. However, because quantitative testing was not associated with improved outcomes in the present study, this could suggest that the more convenient and less expensive qualitative testing is preferred. In practice, however, choice of urinalysis testing method will likely be determined by the purpose and context of the intervention being implemented.

It was noted that exposure to voucher incentive opportunities during Phase 1 did not appear to place participants at an advantage for achieving sustained abstinence during Phase 2. In fact, results suggested that control participants performed somewhat better during the sustained abstinence posttest than those previously exposed to vouchers. Our speculation is that the earning opportunities in Phase 2 may have been more salient for control participants as it was their only opportunity to earn incentives during the entire study. Within the reinforcement conditions there was, however, a strong relationship between abstinence achieved during Phase 1 and Phase 2 on an individual participant level. That is, participants who achieved high rates of continuous cocaine abstinence during the posttest had previously submitted more cocaine-negative urines on both earning and nonearning days during Phase 1 . These observations are consistent with the general principle that past behavior is the best predictor of future behavior, a principle that has been shown to apply to the relationship between during and posttreatment drug abstinence (cf. Higgins, Badger, \& Budney, 2000). Those individuals who submit relatively low rates of cocaine-positive samples prior to the study or during nonreinforced study periods may already be sustaining periods of abstinence similar to those required for reinforcement under the brief abstinence incentive procedures. In this case, relatively little behavior change is required to earn 
reinforcers. One refinement suggested for future studies would be to specifically assess durations of abstinence during periods when incentives are not available and compare these with abstinence durations observed under incentive procedures. This would provide a more refined index of behavior change.

In summary, the present study explored a novel intermittent application of the brief abstinence procedure but found that periodic initiation attempts did not generalize to sustained abstinence under the conditions used. Other methods that more effectively apply principles of intermittent reinforcement schedules (e.g., Petry \& Martin, 2002) may be needed. These data do, however, lend further support to previous demonstrations of the ability of the brief abstinence procedure to promote abstinence initiation and further highlight differences in sensitivity and reinforcement exposure associated with the use of quantitative versus qualitative urine testing methods in abstinence reinforcement procedures.

\section{References}

Grella, C. E., Anglin, M. D., \& Wugalter, S. E. (1997). Patterns and predictors of cocaine and crack use by clients in standard and enhanced methadone maintenance treatment. American Journal of Drug and Alcohol Abuse, 23, 15-42.

Higgins, S. T., Badger, G. J., \& Budney, A. J. (2000). Initial abstinence and success in achieving longer term cocaine abstinence. Experimental and Clinical Psychopharmacology, 8, 377-386.

Higgins, S. T., Budney, A. J., Bickel, W. K., Foerg, F. E., Donham, R., \& Badger, G. J. (1994). Incentives improve outcome in outpatient behavioral treatment of cocaine dependence. Archives of General Psychiatry, 51, 568-576.

Higgins, S. T., Budney, A. J., Bickel, W. K., Hughes, J. R., Foerg, F., \& Badger, G. J. (1993). Achieving cocaine abstinence with a behavioral approach. American Journal of Psychiatry, 150, 763-769.

Higgins, S. T., Delaney, D. D., Budney, A. J., Bickel, W. K., Hughes, J. R., Foerg, F., \& Fenwick, J. W. (1991). A behavioral approach to achieving initial cocaine abstinence. American Journal of Psychiatry, 148, 1218-1224.

Katz, E. C., Robles-Sotelo, E., Correia, C. J., Silverman, K., Stitzer, M. L., \& Bigelow, G. (2002). The brief abstinence test: Effects of continued incentive availability on cocaine abstinence. Experimental and Clinical Psychopharmacology, 10, $10-17$.

Kidorf, M., \& Stitzer, M. L. (1993). Descriptive analysis of cocaine use of methadone patients. Drug and Alcohol Dependence, 32, 267-275.

Petry, N. M., \& Martin, B. (2002). Low-cost contingency management for treating cocaine- and opioid-abusing methadone patients. Journal of Consulting and Clinical Psychology, 70, $398-405$.

Preston, K. L., Silverman, K., Schuster, C. R., \& Cone, E. J. (1997). Assessment of cocaine use with quantitative urinalysis and estimation of new uses. Addiction, 92, 717-727.

Preston, K. L., Umbricht, A., Wong, C. J., \& Epstein, D. H. (2001). Shaping cocaine abstinence by successive approximation. Journal of Consulting and Clinical Psychology, 69, 643-654.

Robles, E., Silverman, K., Preston, K. L., Cone, E. J., Katz, E., Bigelow, G. E., \& Stitzer, M. L. (2000). The brief abstinence test: Voucher-based reinforcement of cocaine abstinence. Drug and Alcohol Dependence, 58, 205-212.

Sidman, M. (1960). Tactics of scientific research. Boston: Authors Cooperative.

Silverman, K., Higgins, S. T., Brooner, R. K., Montoya, I. D., Cone, E. J., Schuster, C. R., \& Preston, K. L. (1996). Sustained cocaine abstinence in methadone maintenance patients through voucher-based reinforcement therapy. Archives of General Psychiatry, 53, 409-415.

Silverman, K., Wong, C. J., Umbricht-Schneither, A., Montoya, I. D., Schuster, C. R., \& Preston, K. L. (1998). Broad beneficial effects of reinforcement for cocaine abstinence in methadone patients. Journal of Consulting and Clinical Psychology, 66, 811-824.

Skinner, B. F. (1953). Science and human behavior. New York: Free Press.

Received September 11, 2003

Revision received June 23, 2004 Accepted June 25, 2004 